\title{
AlleleShift: An R package to predict and visualize population- level changes in allele frequencies in response to climate change
}

\author{
Roeland Kindt ${ }^{\text {Corresp. } 1}$ \\ ${ }^{1}$ World Agroforestry, CIFOR-ICRAF, Nairobi, Kenya \\ Corresponding Author: Roeland Kindt \\ Email address: R.Kindt@CGIAR.org
}

Background. At any particular location, frequencies of alleles that are associated with adaptive traits are expected to change in future climates through local adaption and migration, including assisted migration (human-implemented when climate change is more rapid than natural migration rates). Making the assumption that the baseline frequencies of alleles across environmental gradients can act as a predictor of patterns in changed climates (typically future but possibly paleo-climates), a methodology is provided by AlleleShift of predicting changes in allele frequencies at the population level.

Methods. The prediction procedure involves a first calibration and prediction step through redundancy analysis (RDA), and a second calibration and prediction step through a generalized additive model (GAM) with a binomial family. As such, the procedure is fundamentally different to an alternative approach recently proposed to predict changes in allele frequencies from canonical correspondence analysis (CCA). The RDA step is based on the Euclidean distance that is also the typical distance used in Analysis of Molecular Variance (AMOVA). Because the RDA step or CCA approach sometimes predict negative allele frequencies, the GAM step ensures that allele frequencies are in the range of 0 to 1.

Results. AlleleShift provides data sets with predicted frequencies and several visualization methods to depict the predicted shifts in allele frequencies from baseline to changed climates. These visualizations include 'dot plot' graphics (function shift.dot.ggplot), pie diagrams (shift.pie.ggplot), moon diagrams (shift.moon.ggplot), 'waffle' diagrams (shift.waffle.ggplot) and smoothed surface diagrams of allele frequencies of baseline or future patterns in geographical space (shift.surf.ggplot). As these visualizations were generated through the ggplot2 package, methods of generating animations for a climate change time series are straightforward, as shown in the documentation of AlleleShift and in the supplemental videos.

Availability. AlleleShift is available as an open-source $\mathrm{R}$ package from https://cran.r-project.org/package=AlleleShift and https://github.com/RoelandKindt/AlleleShift . Genetic input data is expected to be in the adegenet::genpop format, which can be generated from the adegenet::genind format. Climate data is available from various resources such as WorldClim and Envirem. 


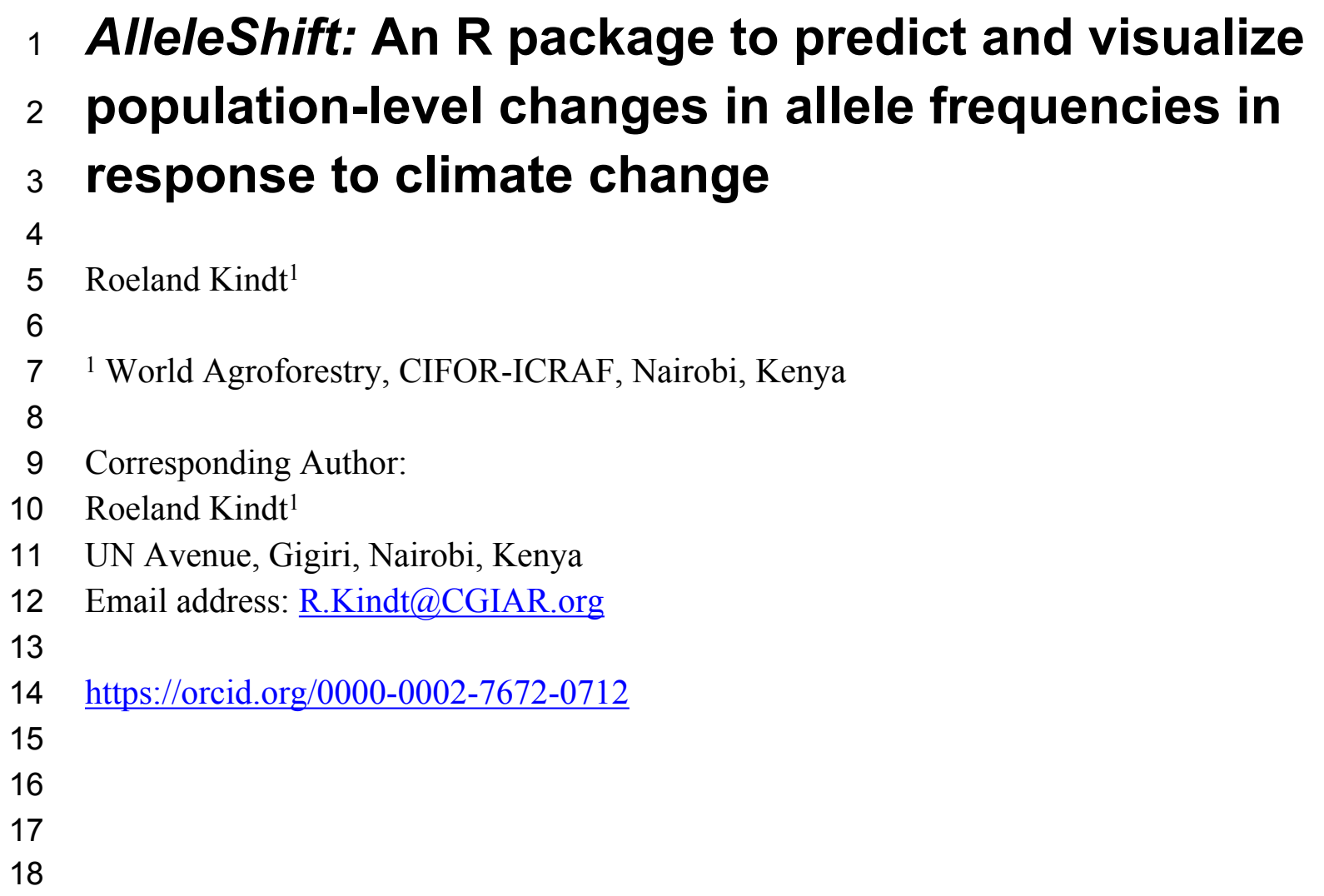




\section{Abstract}

Background. At any particular location, frequencies of alleles that are associated with adaptive traits are expected to change in future climates through local adaption and migration, including assisted migration (human-implemented when climate change is more rapid than natural migration rates). Making the assumption that the baseline frequencies of alleles across environmental gradients can act as a predictor of patterns in changed climates (typically future but possibly paleo-climates), a methodology is provided by AlleleShift of predicting changes in allele frequencies at the population level.

Methods. The prediction procedure involves a first calibration and prediction step through redundancy analysis (RDA), and a second calibration and prediction step through a generalized additive model (GAM) with a binomial family. As such, the procedure is fundamentally different to an alternative approach recently proposed to predict changes in allele frequencies from canonical correspondence analysis (CCA). The RDA step is based on the Euclidean distance that is also the typical distance used in Analysis of Molecular Variance (AMOVA). Because the RDA step or CCA approach sometimes predict negative allele frequencies, the GAM step

Results. AlleleShift provides data sets with predicted frequencies and several visualization methods to depict the predicted shifts in allele frequencies from baseline to changed climates. These visualizations include 'dot plot' graphics (function shift.dot.ggplot), pie diagrams (shift.pie.ggplot), moon diagrams (shift.moon.ggplot), 'waffle' diagrams (shift.waffle.ggplot) and smoothed surface diagrams of allele frequencies of baseline or future patterns in geographical space (shift.surf.ggplot). As these visualizations were generated through the ggplot 2 package, methods of generating animations for a climate change time series are straightforward, as shown in the documentation of AlleleShift and in the supplemental videos.

Availability. AlleleShift is available as an open-source R package from https://cran.rproject.org/package $=$ AlleleShift and https://github.com/RoelandKindt/AlleleShift . Genetic input data is expected to be in the adegenet::genpop format, which can be generated from the adegenet::genind format. Climate data is available from various resources such as WorldClim and Envirem.

\section{Introduction}

There is clear evidence of anthropogenically induced climate change, with our planet facing a climate emergency (Ripple et al., 2020). Anticipating climate change, many countries are developing National Adaptation Plans (NAPs; https:/www4.unfccc.int/sites/napc). Specifically for forests and trees, technical guidelines have recently been prepared on the integration of 
59 forests, agroforestry and trees in the formulation and implementations of NAPs (Meybeck et al.,

60 2020). Stanturf et al. (2015) provide a practical framework and a stoplight tool to plan for

61 climate change mitigation and adaptation in forest and landscape restoration initiatives.

62

63

For loci involved in adaptation, shifts in allele frequencies (and changes in phenotypes as a

64 result) can be anticipated (Günther \& Coop, 2013; Stange, Barrett \& Hendry, 2020). Although the methods involved are far from straightforward, statistical approaches such as Genome Wide Association Studies (GWAS) and Environmental Association Analyses (EAA) can be applied to genomic data to postulate genes and specific alleles involved in climate change responses (Luikart et al., 2018; Anderson \& Song, 2020; Waldvogel et al. 2020). AlleleShift predicts shifts in allele frequencies for those loci predicted by GWAS or EAA to be associated with adaptive traits.

The methodology used in AlleleShift exploits the analogy between analysing a community matrix (consisting of sites as rows, species as columns and abundances as cell values) and a genetic matrix (consisting of populations or individuals as rows, alleles as columns and allele counts as cell values). As a consequence, the common constrained ordination method in the field of community ecology of redundancy analysis (RDA; Legendre \& Legendre 2012, pp. 629 - 661) can be applied.

RDA is based on Euclidean distances and without explanatory variables is equivalent to principal components analysis (PCA). Various recent studies of adaptative genetic variation have also used the RDA methodology (e.g., Razgour et al.; 2019; Capblancq et al., 2020; Nelson, Motamayor \& Cornejo, 2020; Temunović et al., 2020). Analysis of a genetic matrix via Euclidean distances is appropriate for several reasons:

84 - Euclidean distances are also used in Analysis of Molecular Variance (AMOVA; Excoffier, Smouse \& Quattro, 1992; Meirmans \& Liu, 2018; Michalakis \& Excoffier, 1996) and it can be demonstrated (see examples for the AlleleShift: : amova.rda function and possibly also compare with AMOVA analysis in GenAlEx; Peakal \& Smouse, 2012) that RDA provides the same information on squared Euclidean distances and mean squares as an AMOVA analysis. In article S1, I demonstrate how Euclidean distances between adegenet : : genpop objects are linearly related to the Euclidean distances between the centroids obtained from a PCA analysis of adegenet : : genind objects. As a corollary, shifts of populations can be understood as the average shift of individuals in ordination space. Recently, I also showed (Kindt 2020a) how RDA can be directly interpreted in terms of Sums-of-Squares of AMOVA by analysing distances from individuals to centroids and among centroids. 
98

99

100

101

102

103

104

105

106

107

108

109

110

111

112

113

114

115

116

117

118

119

120

121

122

123

124

125

126

127

128

129

130

131

132

133

134

135

136

137

The RDA model is calibrated with an explanatory data set that documents the environmental values of populations in the baseline climate. The prediction function uses the values of populations in the changed (future or past) climate as explanatory variables to predict changes in allele counts and frequencies in the changed climate. As possibly negative allele counts can be predicted by the calibrated RDA, as with the example data set shown below, a second calibration step is implemented that guarantees that allele frequencies are within the biologically realistic interval between 0 and 1 .

By using RDA and avoiding negative allele frequencies, AlleleShift is fundamentally different from the protocol developed by Blumstein et al. (2020) based on canonical correspondence analysis (CCA; Legendre \& Legendre, 20121, pp. 661 - 673). Besides the advantage of RDA to reflect Euclidean distances, a disadvantage of CCA is that the interpretation of species scores in ordination diagrams is more complex in showing the peak of its unimodal distribution against a vector of an explanatory variable (see Fig. 3 in Ter Braak 1987; Fig. 11.9 in Legendre \& Legendre, 2012; or Figure 10.13 in Kindt \& Coe, 2002).

\section{Materials \& Methods}

\section{Data import}

Genetic response data (including a matrix with populations as rows and allele counts as columns) for the calibration of the AlleleShift: : count.model and prediction via AlleleShift: : count.pred is required to be in the adegenet: : genpop format. Individual-based data that are in the adegenet: : genind format can be converted into the genpop format via the adegenet: : genind2genpop function. The adegenet and poppr packages provide various methods of importing data from other software application formats into the genind format, such as adegenet: : import2genind and poppr: :read.genalex. Environmental data of populations, used as explanatory variables in redundancy analysis (RDA), is expected to be provided as a data. frame with the same sequence of populations as the genetic response data (this is a general requirement for community ecology methods in the vegan and Biodiversity $R$ packages; a check is available via

BiodiversityR: : check. datasets). Whereas environmental data typically is baseline and changed (bio)climatic data such as is available from WorldClim (Fick \& Hijmans, 2017), ENVIREM (Title \& Bemmels, 2018), CHELSA (Karger et al., 2020) or PaleoClim (Brown et al., 2018), it is also possible to expand input to other data available for species distribution modelling (a recent overview of available data sets is provided by Booth, 2018).

\section{Data analysis}

Prior to calibrating the models that predict allele frequencies from bioclimatic explanatory data, it is recommended to reduce the explanatory variables to a subset where the Variance Inflation Factor (VIF) is below a predefined threshold for each variable. Such methods are also 
138

139

140

141

142

143

144

145

146

147

148

149

150

151

152

153

154

155

156

157

158

159

160

161

162

163

164

165

166

167

168

169

170

171

172

173

174

175

176

177

recommended for regression analysis (Fox \& Monette, 1992) and species distribution modelling (Kindt, 2018). With this approach, it is easy to select the same variables from future data sets and comparison with other studies may also become easier. VIF analysis, and an additional feature of forcing the algorithm to keep preselected variables within the final subset, is available via AlleleShift: :VIF. subset (Table 1). This is a function that uses

BiodiversityR: : ensemble.VIF. dataframe internally after a first step of removing all explanatory variables that have correlations larger than the VIF threshold with the preselected variables. There is an option to generate a correlation matrix for the final subset of variables (Figure 1). I also recommend conducting the VIF analysis for the genetic response data (see discussion and Figure 1). I further advise to remove any individuals with partially missing genetic or (bio)climatic data prior to the analysis. As a sensitivity analysis, results could possibly be compared with models calibrated with data where missing genotypes were replaced by genotypes predicted by the impute function of the LEA package (Gain \& François, 2021).

Prior to model calibration, I suggest checking (according to criteria described below) for the shifts of populations in environmental space between the baseline and changed climates. This can be achieved via function AlleleShift: population.shift, which draws arrows between each population in the baseline and changed climate. There are alternatives of using principal components analysis (PCA) or redundancy analysis (RDA) to generate the ordination diagrams (Figure 2). What is important to check in the ordination graphs is whether populations shift in a similar fashion, as that will facilitate the interpretation of predicted shifts in allele frequencies. If some populations would show a different trend in the ordination graph, their allele frequencies would also be expected to change in a different way.

With the selected genetic and explanatory data, model calibration can be done. In a first step, a RDA model is fitted (AlleleShift: : count.model) that can predict counts of alleles in baseline or changed climates (AlleleShift: : count.pred). The user has the option also to obtain results for the canonical correspondence analysis procedure of Blumstein et al. (2020) with the count model via argument $\mathrm{cca}$. model=TRUE. In the second step, the predicted allele counts for the baseline climate serve as explanatory variables for the calibration of a generalized linear model (GAM via mgcv : : gam; Wood, 2004) with the baseline allele frequencies as response and a binomial family function (AlleleShift: : freq. model). This procedure ensures that predictions are within the realistic interval for frequencies between 0 to 1 . Function AlleleShift: : freq.pred allows the prediction of allele frequencies for baseline and changed climates. A simulation study (article S2, using a subset of a simulation study available from Frichot \& François, 2015) and the results below show that the predicted frequencies approximate the expected frequencies well.

The output of the two steps (RDA followed by GAM) is a data.frame as shown in Table 2 (to fit printing space available here, the data is shown in a transposed format where rows and columns 
178 were swapped). All figures shown in this manuscript were obtained with the example data sets of 179 AlleleShift: : Poptri.genind (individually-based allele counts),

180 AlleleShift: : Poptri.baseline.env (climatic descriptors of the populations in the 181 baseline climate), AlleleShift: : Poptri.future. env (climatic descriptors of the 182 populations in the future climate) and AlleleShift: : Poptri.loc (geographical

183 coordinates of the populations). These data sets were converted from the example data sets 184 provided by Blumstein et al. (2020) for Populus trichocarpa. It can be seen for population 185 Nisqually in our case study that negative allele counts and frequencies are predicted for one of 186 the minor alleles in the RDA prediction step, but that the GAM step predicts the biologically

187

188 189

190

191

192

193

194

195

196

197

198

199

200

201

202

203

204

205

206

207

208

209

210

211

212

213

214

215

216

217 acceptable frequency of 0.027. Function Alleleshift: : freq.ggplot (Figure 3) enables a visual inspection of the power of the models to predict allele frequencies for the calibration data.

\section{Visualizations}

AlleleShift generates various types of ggplot2 (Wickham, 2016; version 3.3.2) graphics from the output of AlleleShift: : freq.pred. These include dot (Figure 4), pie or donut (Figure 5), moon (Figure 6) and waffle (Figure 7) graphics, and smoothed regression surfaces (Figure 8). As an intermediate step to generate various of these graphics, helper functions such as waffle.baker for shift.waffle.ggplot or moon. waxer for shift.moon.ggplot prepare data for the main graphing function (Table 1). With default settings, visualizations depict changes in allele frequencies for each allele in different panels, internally via ggplot $2:$ : facet_grid. Setting argument mean. change to TRUE, the graphics depict median or mean changes in allele frequencies.

Function shift.surf.ggplot plots populations in geographical space via their geographical coordinates (longitude and latitude in Figure 8). The function fits a smoothed regression surface for allele frequencies via vegan: : ordisurf. This output is then further processed internally within the function via BiodiversityR: : ordisurfgrid.long (an overview of generating ggplot2 ordination diagrams via vegan and BiodiversityR is given in Kindt, 2020b; these guidelines can be used to customize ordination graphs as shown in Figures 2 and 8). Various options of fitting smoothed regression surfaces are available by providing additional arguments to shift.surf.ggplot, such as the various mgcv: : smooth.terms options of thin plates, Duchon splines, cubic regression splines, P-splines, Markov Random Fields, Gaussian process smooths, soap film smooths, splines on the sphere and random effects.

As graphics are generated with ggplot2, it is relatively easy to generate animated versions of visualizations with gganimate (Pedersen \& Robinson, 2020; version 1.0.7). Scripts for generating animated versions for dot graphics, pie graphics and smoothed surfaces are provided with the documentation of the respective functions. Supplemental MP4 videos (S3 - S5) are for a time series that interpolates bioclimatic data between baseline and future climate in steps of five 
218 years; these also extrapolate data far into the future (up to a million years, see the Results and 219 Discussion for my reason to do this). Other than time series, animated visualizations could also 220 be generated for different global circulation models (GCMs) or scenarios such as various shared 221 socio-economic pathways (SSPs) developed in the context of the sixth assessment report of the 222 Intergovernmental Panel on Climate Change .

223

224

225

226

227

228

229

230

231

232

233

234

235

236

237

238

239

240

241

242

243

244

245

246

247

248

249

250

251

252

253

254

255

\section{Results and Discussion}

AlleleShift predicts shifts in allele frequencies via RDA and GAM, an alternative pathway that maintains Euclidean distances among populations and individuals (Article S1). It also avoids making negative frequency predictions as what may occur with the protocol recently devised by Blumstein et al. (2020). My methodology however faces the same limitations of data requirements as discussed by Blumstein et al. (2020) for their protocol in terms of the initial identification of responsive markers. Key assumptions of the Blumstein et al. (2020) protocol apply also to my approach, including that allelic effects are independent and additive with no epistasis or dominance (see also the discussion on epistasis, structural genomic variation and epigenetics by Stange, Barrett \& Hendry, 2020).

I recommend reducing both the explanatory variables and the response variables to subsets of data with a maximum Variance Inflation Factor (VIF) of 20 or less (Ter Braak \& Smilauer, 2002), for alleles to obtain better estimates of changes in their frequencies. For the allele counts as response variables, if VIF would be larger than 20, I would use function VIF . subset in an iterative procedure whereby earlier selected subsets of alleles are excluded, to generate different subsets of alleles (but keeping variables for both $\mathrm{A}$ and $\mathrm{B}$ allele counts in each final subset). For example, with 20 alleles X01A, X01B, X02A, X02B, .., X10A, X10B, the two subsets could be $\{\mathrm{X} 01 \mathrm{~A}, \mathrm{X} 01 \mathrm{~B}, \ldots, \mathrm{X} 05 \mathrm{~A}, \mathrm{X} 05 \mathrm{~B}\}$ and $\{\mathrm{X} 06 \mathrm{~A}, \mathrm{X} 06 \mathrm{~B}, \ldots, \mathrm{X} 10 \mathrm{~A}, \mathrm{X} 10 \mathrm{~B}\}$. With the various subsets, shifts in allele frequencies can then be predicted, and finally predictions with all subsets can be combined.

When predictions are made by AlleleShift into the future, and especially into novel climatic conditions, it is warranted to consider the transferability of the calibrated models and ideally to provide 'transferability metrics' that quantify prediction uncertainty (Yates et al., 2018). The function of environmental. novel identifies populations that have novel climatic conditions (values outside the baseline range) for at least one of the explanatory variables for the changed climate. This function also calculates the probabilities of encountering the novel conditions from the mean and standard deviation of the baseline ranges and then returns the lowest of these probabilities as an indicator of the degree of novelty of the environmental conditions. 
257 For the animated graphics, as an extreme example of the caveats of the methodology, I made

258

259

260

261

262

263

264

265

266

267

268

269

270

271

272

273

274

275

276

277

278

279

280

281

282

283

284

285

286

287

288

289

290

291

292

293

294

295

296

projections one million years into the future. These projections followed earlier trends of the $21^{\text {st }}$ century and resulted in allele frequencies becoming fixed at 0 or 1 , which is a biologically possible scenario. At the same time, however, these simulations clearly illustrated that the methodology through RDA is correlative as in correlative/phenomenological species distribution models. In fact, the linear extrapolation of climate variables resulted in an environmental data set where the mean annual temperature was above 30,000 degrees, which obviously is a biologically irrelevant scenario. Thus, the method presented here should be used cautiously in novel climates especially as predictions will be available (the model will not crash or give an error warning), and should thus be used even more cautiously where differences between baseline and future climates are large.

As an approach to obtain a better handle on the transferability of AlleleShift predictions into future climates, I recommend to also estimate the habitat suitability via species distribution models (SDMs). Where habitat suitability is below a certain threshold, for instance a threshold where the sum of sensitivity and specificity is maximized (Liu, White \& Newell, 2013), results of predictions of AlleleShift could be annotated of having lower transferability. Similarly, where evaluation strips as proposed by Elith et al. (2005) suggest that it is less likely that the habitat is suitable within the changed range of environmental conditions, additional information could be provided on a lower transferability. Well-documented methods of utilizing SDMs to predict shifts in species habitat suitability are available in the literature, including recent examples that use the ensemble suitability modelling framework available in Biodiversity $R$ (e.g., de Sousa et al., 2019; Fremout et al., 2020; Kindt, 2018; Ranjitkar et al., 2014). For organisms such as trees, correlative SDMs remain the best available method of predicting future species suitability, whereas the limitations of these methods may not be as great as has been suggested (Booth, 2016; Booth, 2018). What is also attractive about SDMs is that a wider set of presence observations are likely to be available than those populations that have been studied genetically. Presence data are available from open-source databases such as GBIF or the Botanical Information and Ecology Network (Enquist et al., 2016). Further to the collation of a larger set of presence observations, application of SDMs should be straightforward using the same (bio)climatic data sets as applied in AlleleShift. The approach of combining the results of AlleleShift with SDM is somewhat similar to the method applied by Aguirre-Liguori et al. (2019) to develop species distribution models for alleles. In my proposal, however, the predictions of allele frequencies and SDM are done independently, and ideally with an expanded point presence data set for SDM.

A straightforward and practical expansion of the methodology I have proposed is to tree seed sourcing programmes (Broadhurst et al., 2008), possibly for developing schemes of humanassisted geneflow sensu Aitken \& Whitlock (2013). This is important for ensuring the matching of planting materials to the conditions of planting sites (Cernansky, 2018; Roshetko et al., 2018;

Peer) reviewing PDF | (2021:01:57565:1:0:NEW 8 Apr 2021) 
297 Kettle et al., 2020). For specific planting sites and planting times (considering the perennial

298

299

300

301

302

303

304

305

306

307

308

309

310

311

312

313

314

315

316

317

318

319

320

321

322

323

324

325

326

327

328

329

330

331

332

333

334

nature of trees, climate change during the production cycle should be considered) of interest, the prediction methods can readily provide the predicted allele frequencies needed for adaptation.

Theoretically, based on the similarity between predicted allele frequencies and those of available source populations, the best matching source can then be selected. Similar approaches to devise transfer and conservation schemes in the face of climate change can be employed for other organisms than plants (Fitzpatrick \& Edelsparre, 2018; Rochat et al., 2021).

\section{Conclusions}

The R package AlleleShift provides a set of functions that allow the prediction of allele frequencies from baseline, future and past (bio)climatic explanatory variables via redundancy analysis (RDA) and generalized additive models (GAM). Various visualizations are provided via ggplot2 and its extension packages such as ggforce and gganimate. At the time of submission of this manuscript, no package was available for this set of tools. As with any other methodology that projects into the future, it is important to reflect on transferability to novel climates.

\section{Acknowledgements}

The author thanks Ian Dawson (CIFOR-ICRAF) for reviewing the article prior to submission and three anonymous reviewers who provided excellent suggestions to improve the manuscript. He also thanks additional colleagues from CIFOR-ICRAF and the University of Copenhagen for useful discussions on the applications of this package, including Lars Graudal, Prasad Hendre, Ramni Jamnadass and Jens-Peter B. Lillesø. The CGIAR Research Program on Forests, Trees, and Agroforestry (supported by the CGIAR Trust Fund) and the Provision of Adequate Tree Seed Portfolios project (supported by Norway's International Climate and Forest Initiative through the Royal Norwegian Embassy in Ethiopia) supported the author's time on this project.

\section{References}

Aguirre-Liguori, J.A., Ramírez-Barahona, S., Tiffin, P. and Eguiarte, L.E., 2019. Climate change is predicted to disrupt patterns of local adaptation in wild and cultivated maize.

Proceedings of the Royal Society B, 286(1906), p.20190486. https://doi.org/10.1098/rspb.2019.0486

Aitken, S.N., Whitlock, M.C. (2013). Assisted gene flow to facilitate local adaptation to climate change. Annual review of ecology, evolution, and systematics, 44. https://doi.org/10.1146/annurev-ecolsys-110512-135747

Anderson, J.T. and Song, B.-H. (2020). Plant adaptation to climate change-Where are we? J. Syst. Evol., 58: 533-545. https://doi.org/10.1111/jse.12649

Blumstein et al. 2020. Protocol for Projecting Allele Frequency Change under Future Climate Change at Adaptive-Associated Loci. https://doi.org/10.1016/j.xpro.2020.100061 
335

336

337

338

339

340

341

342

343

344

345

346

347

348

349

350

351

352

353

354

355

356

357

358

359

360

361

362

363

364

365

366

367

368

369

370

371

372

373

374

Booth, T.H. (2016). Estimating potential range and hence climatic adaptability in selected tree species. Forest Ecology and Management, 366, pp.175-183. https://doi.org/10.1016/j.foreco.2016.02.009

Booth, T.H. (2018). Species distribution modelling tools and databases to assist managing forests under climate change. Forest Ecology and Management 430: 196-203. URL https://doi.org/10.1016/j.foreco.2018.08.019.

Bramson, M. (2019). gggibbous: Moon Charts, a Pie Chart Alternative. R package version 0.1.0. https://CRAN.R-project.org/package=gggibbous

Broadhurst, L.M., Lowe, A., Coates, D.J., Cunningham, S.A., McDonald, M., Vesk, P.A. and Yates, C. (2008), Seed supply for broadscale restoration: maximizing evolutionary potential. Evolutionary Applications, 1: 587-597. https://doi.org/10.1111/j.17524571.2008.00045.x

Brown, J., Hill, D., Dolan, A. et al. (2018). PaleoClim, high spatial resolution paleoclimate surfaces for global land areas. Sci Data 5, 180254. https://doi.org/10.1038/sdata.2018.254

Capblancq, T, Morin, X, Gueguen, M, Renaud, J, Lobreaux, S, Bazin, E. Climate-associated genetic variation in Fagus sylvatica and potential responses to climate change in the French Alps. J Evol Biol. 2020; 33: 783- 796. https://doi.org/10.1111/jeb.13610

Cernansky, R. (2018). How to plant a trillion trees. Nature 560: 542-544. https://doi.org/10.1038/d41586-018-06031-x

de Sousa, K., van Zonneveld, M., Holmgren, M. et al. (2019). The future of coffee and cocoa agroforestry in a warmer Mesoamerica. Sci Rep 9, 8828. https://doi.org/10.1038/s41598019-45491-7

Elith, J., Ferrier, S., Huettmann, F. and Leathwick, J., 2005. The evaluation strip: a new and robust method for plotting predicted responses from species distribution models. Ecological modelling, 186(3), pp.280-289. https://doi.org/10.1016/j.ecolmodel.2004.12.007

Enquist, B.J., Condit, R., Peet, R.K., Schildhauer, M. and Thiers, B.M., 2016. Cyberinfrastructure for an integrated botanical information network to investigate the ecological impacts of global climate change on plant biodiversity (No. e2615v2). PeerJ Preprints. https://doi.org/10.7287/peerj.preprints.2615v2

Excoffier, L., Smouse, P.E. and Quattro, J.M. (1992). Analysis of molecular variance inferred from metric distances among DNA haplotypes: application to human mitochondrial DNA restriction data. Genetics, 131, 479-491.

Fick, S.E., Hijmans, R.J. (2017). WorldClim 2: new $1 \mathrm{~km}$ spatial resolution climate surfaces for global land areas. International Journal of Climatology 37 (12): 4302-4315. https://www.worldclim.org

Fitzpatrick, M.J. and Edelsparre, A.H., 2018. The genomics of climate change. Science, 359(6371), pp.29-30. https://science.sciencemag.org/content/359/6371/29.full

Fox, J., Monette, G., 1992. Generalized collinearity diagnostics. J. Am. Stat. Assoc. 87, 178e183. https://doi.org/10.1080/01621459.1992.10475190. 
375 Fremout, T, Thomas, E, Gaisberger, H, et al. (2020). Mapping tree species vulnerability to

376

377

378

379

380

381

382

383

384

385

386

387

388

389

390

391

392

393

394

395

396

397

398

399

400

401

402

403

404

405

406

407

408

409

410

411

412

413

414 multiple threats as a guide to restoration and conservation of tropical dry forests. Glob Change Biol.; 26: 3552- 3568. https://doi.org/10.1111/gcb.15028

Frichot, E. and François, O. (2015), LEA: An R package for landscape and ecological association studies. Methods Ecol Evol, 6: 925-929. https://doi.org/10.1111/2041210X.12382

Gain, C. and François, O. (2021), LEA 3: Factor models in population genetics and ecological genomics with R. Mol Ecol Resour. https://doi.org/10.1111/1755-0998.13366.

Günther, T. and Coop, G., 2013. Robust identification of local adaptation from allele frequencies. Genetics, 195(1), pp.205-220. https://dx.doi.org/10.1534\%2Fgenetics.113.152462

Kamvar ZN, Tabima JF, Grünwald NJ. (2014) Poppr: an R package for genetic analysis of populations with clonal, partially clonal, and/or sexual reproduction. PeerJ 2:e281. https://doi.org/10.7717/peerj.281

Karger, D.N., Schmatz, D.R., Dettling, G. et al. High-resolution monthly precipitation and temperature time series from 2006 to 2100. Sci Data 7, 248 (2020). https://doi.org/10.1038/s41597-020-00587-y

Kettle, C.J., Atkinson, R., Boshier, D., Ducci, F., Dawson, I., Ekué, M., Elias, M., Graudal, L., Jalonen, R., Koskela, J. and Monteverdi, M.C., 2020. Priorities, challenges and opportunities for supplying tree genetic resources. Restoring the Earth-The next decade: Unasylva No. 252-Vol. 71 2020/1, 252(1), p.51. http://www.fao.org/3/cb1600en/CB1600EN.pdf

Kindt, R. (2018). Ensemble species distribution modelling with transformed suitability values. Environmental Modelling \& Software 100: 136-145. https://doi.org/10.1016/j.envsoft.2017.11.009

Kindt, R. (2020a). Ordination graphs with vegan, BiodiversityR and ggplot2. https://rpubs.com/Roeland-KINDT

Kindt, R. (2020b). Analysis of Molecular Variance (AMOVA) with vegan and BiodiversityR, including a graphical method to identify potential migrants. https://rpubs.com/RoelandKINDT

Kindt, R., Coe, R. (2005). Tree Diversity Analysis. A manual and software for common statistical methods for ecological and biodiversity studies. https://CRAN.Rproject.org/package $=$ BiodiversityR

Lasky, J.R., Forester, B.R. and Reimherr, M., 2018. Coherent synthesis of genomic associations with phenotypes and home environments. Molecular Ecology Resources, 18(1), pp.91106. https://doi.org/10.1111/1755-0998.12714

Legendre, P., Legendre, L., 2012. Numerical ecology. Elsevier.

Liu, C., White, M. and Newell, G. (2013), Selecting thresholds for the prediction of species occurrence with presence-only data. J. Biogeogr., 40: 778-789. https://doi.org/10.1111/jbi.12058 
415 Luikart G., Kardos M., Hand B.K., Rajora O.P., Aitken S.N., Hohenlohe P.A. (2018) Population

416

417

418

419

420

421

422

423

424

425

426

427

428

429

430

431

432

433

434

435

436

437

438

439

440

441

442

443

444

445

446

447

448

449

450

451

452

453

454 Genomics: Advancing Understanding of Nature. In: Rajora O. (eds) Population Genomics. Population Genomics. Springer, Cham. https://doi.org/10.1007/13836_2018_60

Meirmans, P., Liu, S. (2018). Analysis of Molecular Variance (AMOVA) for Autopolyploids Front. Ecol. Evol., 23. https://doi.org/10.3389/fevo.2018.00066

Meybeck, A., Gitz, V., Wolf, J. and Wong, T. 2020. Addressing forestry and agroforestry in National Adaptation Plans - Supplementary guidelines. Place of publication, Bogor/Rome. FAO and FTA. https://doi.org/10.4060/cb1203en

Michalakis, Y., Excoffier, L. (1996). A generic estimation of population subdivision using distances between alleles with special reference for microsatellite loci. Genetics 142, 1061-1064.

Nelson, J.T., Motamayor, J.C. and Cornejo, O.E., 2020. Environment and pathogens shape local and regional adaptations to climate change in the chocolate tree, Theobroma cacao L. Molecular Ecology. https://doi.org/10.1111/mec.15754

Peakall R and Smouse PE. 2012. GenAlEx 6.5: genetic analysis in Excel. Population genetic software for teaching and research - an update. https://doi.org/10.1093/bioinformatics/bts460.

Pedersen, T.L. (2020). ggforce: Accelerating 'ggplot2'. R package version 0.3.2. https://CRAN.R-project.org/package $=$ ggforce

Pedersen, T.L., Robinson, D. (2020). gganimate: A Grammar of Animated Graphics. R package version 1.0.7. https://CRAN.R-project.org/package=gganimate

Ranjitkar, S., Xu, J., Shrestha, K.K. and Kindt, R. (2014). Ensemble forecast of climate suitability for the Trans-Himalayan Nyctaginaceae species. Ecological Modelling, 282: 824. https://doi.org/10.1016/j.ecolmodel.2014.03.003

Razgour, O., Forester, B., Taggart, J.B., Bekaert, M., Juste, J., Ibáñez, C., Puechmaille, S.J., Novella-Fernandez, R., Alberdi, A. and Manel, S. (2019). Considering adaptive genetic variation in climate change vulnerability assessment reduces species range loss projections. Proceedings of the National Academy of Sciences, 116(21), 10418-10423. https://doi.org/10.1073/pnas.1820663116

Rengefors, K., Gollnisch, R., Sassenhagen, I., Härnström Aloisi, K., Svensson, M., Lebret, K., Čertnerová, D., Cresko, W.A., Bassham, S. and Ahrén, D. (2021). Genome-wide SNP markers reveal population structure and dispersal direction of an expanding nuisance algal bloom species. Molecular Ecology. https://doi.org/10.1111/mec.15787

Ripple, W.J., Christopher Wolf, Thomas M Newsome, Phoebe Barnard, William R Moomaw, et al. (2020). World Scientists' Warning of a Climate Emergency, BioScience 70: 8-12, https://doi.org/10.1093/biosci/biz088

Rochat, E, Selmoni, O, Joost, S. (2021). Spatial areas of genotype probability: Predicting the spatial distribution of adaptive genetic variants under future climatic conditions. Divers Distrib. 00: 1- 15. https://doi.org/10.1111/ddi.13256

Peer] reviewing PDF | (2021:01:57565:1:0:NEW 8 Apr 2021) 
455

456

457

458

459

460

461

462

463

464

465

466

467

468

469

470

471

472

473

474

475

476

477

478

479

480

481

482

483

484

485

486

487

488

489

490

491

Roshetko, J.M., Dawson, I.K., Urquiola, J., Lasco, R.D., Leimona, B., Weber, J.C., Bozzano, M., Lillesø, J.P.B., Graudal, L. and Jamnadass, R. (2018). To what extent are genetic resources considered in environmental service provision? A case study based on trees and carbon sequestration. Climate and Development, 10(8), pp.755-768. https://doi.org/10.1080/17565529.2017.1334620

Stange, M., Barrett, R.D. and Hendry, A.P. (2020). The importance of genomic variation for biodiversity, ecosystems and people. Nature Reviews Genetics, pp.1-17.

Stanturf, J.A, Kant, P., Lillesø, J.-P.B., Mansourian, S., Kleine, M., Lars Graudal, L., Palle Madsen, P. (2015). Forest Landscape Restoration as a Key Component of Climate Change Mitigation and Adaptation. IUFRO World Series Volume 34. Vienna 72 p.

Temunović, M., Garnier-Géré, P., Morić, M., Franjić, J., Ivanković, M., Bogdan, S. and Hampe, A. (2020). Candidate gene SNP variation in floodplain populations of pedunculate oak (Quercus robur L.) near the species' southern range margin: Weak differentiation yet distinct associations with water availability. Molecular Ecology, 29(13), pp.2359-2378. https://doi.org/10.1111/mec.15492

Ter Braak, C.J. and Smilauer, P., 2002. CANOCO reference manual and CanoDraw for Windows user's guide: software for canonical community ordination (version 4.5).

Ter Braak, C.J., 1987. The analysis of vegetation-environment relationships by canonical correspondence analysis. Vegetatio, 69(1), pp.69-77.

Title P.O., Bemmels J.B. (2018). ENVIREM: an expanded set of bioclimatic and topographic variables increases flexibility and improves performance of ecological niche modeling. Ecography. 41:291-307. https://envirem.github.io/

Waldvogel, A.-M., Feldmeyer, B., Rolshausen, G., Exposito-Alonso, M., Rellstab, C., Kofler, R., Mock, T., Schmid, K., Schmitt, I., Bataillon, T., Savolainen, O., Bergland, A., Flatt, T., Guillaume, F. and Pfenninger, M. (2020), Evolutionary genomics can improve prediction of species' responses to climate change. Evolution Letters, 4: 4-18. https://doi.org/10.1002/ev13.154

Wickham, H. (2016). ggplot2: Elegant Graphics for Data Analysis. Springer-Verlag New York, 2016. https://ggplot2.tidyverse.org

Wood, S.N. (2004) Stable and Efficient Multiple Smoothing Parameter Estimation for Generalized Additive Models, Journal of the American Statistical Association, 99: 467, 673-686. https://doi.org/10.1198/016214504000000980

Yates, K.L., Phil J. Bouchet, P.J., Caley, M.J., Mengersen, K., Randin, C.F., Parnell, S., Fielding, A.H., Bamford, A.J., Ban, S., et al. (2018). Outstanding Challenges in the Transferability of Ecological Models. Trends in Ecology \& Evolution 33: 790-802. URL https://doi.org/10.1016/j.tree.2018.08.001. 


\section{Table $\mathbf{1}$ (on next page)}

Functions found in AlleleShift and their short description. 
1 Table 1. Functions found in AlleleShift and their short descriptions

\begin{tabular}{|c|c|}
\hline Function & Description \\
\hline \multicolumn{2}{|l|}{ Preparation } \\
\hline VIF.subset & $\begin{array}{l}\text { Reduce the number of explanatory variables through Variance } \\
\text { Inflation Factor analysis, with an option to plot a correlation } \\
\text { matrix (GGlly: : ggcorr). Internally, the function calls the } \\
\text { BiodiversityR: : ensemble.VIF. dataframe } \\
\text { function. }\end{array}$ \\
\hline environmental.novel & $\begin{array}{l}\text { Identify populations with some values of the explanatory } \\
\text { variables that are outside the range of values used for } \\
\text { calibration. }\end{array}$ \\
\hline \multicolumn{2}{|l|}{ Analysis } \\
\hline count.model & $\begin{array}{l}\text { Calibration of RDA model (vegan : : rda) with baseline allele } \\
\text { counts as response and baseline bioclimatic variables as } \\
\text { explanatory variables }\end{array}$ \\
\hline count.pred & $\begin{array}{l}\text { Prediction of allele counts (vegan: : predict.rda) from } \\
\text { explanatory variables. Explanatory variables correspond to the } \\
\text { baseline climate to check the calibration }\end{array}$ \\
\hline freq.model & $\begin{array}{l}\text { Calibration of GAM model (mgcv: : gam) with baseline allele } \\
\text { frequencies as response and predicted baseline counts from the } \\
\text { RDA model as explanatory variables }\end{array}$ \\
\hline freq.pred & $\begin{array}{l}\text { Prediction of allele frequencies (mgcv: : predict.gam) } \\
\text { from the predicted alleles counts of count.pred }\end{array}$ \\
\hline amova.rda & $\begin{array}{l}\text { Perform AMOVA with the outputs from RDA. The function } \\
\text { returns an output that is similar to the output of } \\
\text { poppr: : poppr.amova so that results can be readily } \\
\text { compared }\end{array}$ \\
\hline \multicolumn{2}{|l|}{ Visualization } \\
\hline population.shift & $\begin{array}{l}\text { Shifts of populations in environmental space, with superellipses } \\
\text { (ggforce: : geom_mark_ellipse) and arrows between } \\
\text { baseline and changed positions to show climatic shifts. } \\
\text { Internally, the function calls vegan::ordiplot and various helper } \\
\text { functions from BiodiversityR (Kindt 2020a) are used }\end{array}$ \\
\hline freq.ggplot & $\begin{array}{l}\text { Plots of baseline allele frequencies against predicted allele } \\
\text { frequencies. Data points can be coloured differently by } \\
\text { population or by allele }\end{array}$ \\
\hline shift.dot.ggplot & Shifts of Allele Frequencies as Response to Climate Change \\
\hline shift.pie.ggplot & $\begin{array}{l}\text { Shifts of Allele Frequencies as Response to Climate Change via } \\
\text { ggforce: : geom_arc_bar }\end{array}$ \\
\hline pie.baker & $\begin{array}{l}\text { Helper function to prepare data for shift.pie.ggplot } \\
\text { from the output of freq.pred. }\end{array}$ \\
\hline shift.moon.ggplot & $\begin{array}{l}\text { Shifts of Allele Frequencies as Response to Climate Change via } \\
\text { gggibbous: : geom_moon }\end{array}$ \\
\hline moon.waxer & $\begin{array}{l}\text { Helper function to prepare data for shift.moon.ggplot } \\
\text { from the output of freq.pred }\end{array}$ \\
\hline
\end{tabular}




\begin{tabular}{|l|l|}
\hline Function & Description \\
\hline shift.waffle.ggplot & $\begin{array}{l}\text { Shifts of Allele Frequencies as Response to Climate Change. } \\
\text { Graphics are similar to waffle: :waffle, but the graph is } \\
\text { made de novo in AlleleShift }\end{array}$ \\
\hline waffle.baker & $\begin{array}{l}\text { Helper function to prepare data for shift.waffle.ggplot } \\
\text { from the output of freq.pred }\end{array}$ \\
\hline shift.surf.ggplot & $\begin{array}{l}\text { Shifts of Allele Frequencies as Response to Climate Change, } \\
\text { plotted in geographical space through smoothed regression } \\
\text { surfaces (vegan: : ordisurf) }\end{array}$ \\
\hline
\end{tabular}

\section{Notes}

3 BiodiversityR (Kindt \& Coe, 2005; version 2.12-3 used in this manuscript); ggforce (Pedersen, 4 2000; version 0.3.2); gggibbous (Bramson 2019; version 0.1.0); mgcv (Wood, 2004; version 1.85 31); poppr (Kamvar, Tabima \& Grünwald, 2014; version 2.8.6); vegan (Oksanen et al., 2020; 6 version 2.5-6). 


\section{Table 2 (on next page)}

Output from function freq.pred for the changed climate for four populations and 2 alleles ( $A$ and $B)$.

This is a subset of the result with all populations and all alleles for the example data sets in AlleleShift for Populus trichocarpa. 
1 Table 2. Output from function freq. pred for the changed climate for four populations and 2

2 alleles (A and B). This is a subset of the result with all populations and all alleles for the example

3 data sets in AlleleShift for Populus trichocarpa.

4

\begin{tabular}{|c|c|c|c|c|}
\hline Population & Puyallup & Tahoe & Skagit & Nisqually \\
\hline $\mathrm{N}$ & 372 & 24 & 326 & 28 \\
\hline Locus & \multicolumn{4}{|c|}{ X01_10838495 } \\
\hline Allele.freq & 0.172 & 0.625 & 0.236 & 0.107 \\
\hline $\mathrm{A}$ & 64 & 15 & 77 & 3 \\
\hline $\mathrm{B}$ & 308 & 9 & 249 & 25 \\
\hline Ap & 144.376 & 23.471 & 75.381 & 15.165 \\
\hline $\mathrm{Bp}$ & 227.624 & 0.529 & 250.619 & 12.835 \\
\hline N.e1 & 372 & 24 & 326 & 28 \\
\hline Freq.e1 & 0.388 & 0.978 & 0.231 & 0.542 \\
\hline Freq.e2 & 0.425 & 0.959 & 0.192 & 0.633 \\
\hline $\mathrm{LCL}$ & 0.306 & 0.794 & 0.175 & 0.452 \\
\hline UCL & 0.544 & 1.000 & 0.210 & 0.813 \\
\hline increasing & TRUE & TRUE & FALSE & TRUE \\
\hline Locus & \multicolumn{4}{|c|}{ X01_16628872 } \\
\hline Allele.freq & 0.169 & $0.0 \overline{8}$ & 0.181 & 0.250 \\
\hline $\mathrm{A}$ & 63 & 2 & 59 & 7 \\
\hline $\mathrm{B}$ & 309 & 22 & 267 & 21 \\
\hline $\mathrm{Ap}$ & 38.751 & 0.509 & 52.668 & -0.038 \\
\hline $\mathrm{Bp}$ & 333.249 & 23.491 & 273.332 & 28.038 \\
\hline N.e1 & 372 & 24 & 326 & 28 \\
\hline Freq.e1 & 0.104 & 0.021 & 0.162 & -0.001 \\
\hline Freq.e2 & 0.104 & 0.037 & 0.183 & 0.027 \\
\hline $\mathrm{LCL}$ & 0.091 & 0.029 & 0.161 & 0.018 \\
\hline UCL & 0.117 & 0.045 & 0.205 & 0.036 \\
\hline increasing & FALSE & FALSE & TRUE & FALSE \\
\hline
\end{tabular}

5 Notes

$6 \mathrm{~N}$, number of alleles sampled from population; Allele.freq, frequency of the minor allele in the

7 baseline climate calculated via a degenet: : make. freq; $\mathrm{A} / \mathrm{B}$, count of the alleles in the

8 baseline climate; Ap/Bp, predicted counts of the alleles in the changed climate; N.e1, predicted

9 number of alleles; Freq.e1: predicted frequency of the minor allele via RDA; Freq.e2, predicted

10 frequency of the minor allele via GAM; LCL/UCL: lower/ upper confidence limits; increasing,

11 flag whether frequency is increasing. 


\section{Figure 1}

Correlation matrices.

(A) Correlation matrix plot for the final subset of bioclimatic variables selected by the VIF.subset function. CMI: climatic moisture index, Eref: reference atmospheric evaporative demand, SHM: summer heat-to-moisture index, AHM: annual heat-to-moisture index, TD: continentality, MAT: mean annual temperature. (B) Correlation matrix plot for the minor alleles. 
A

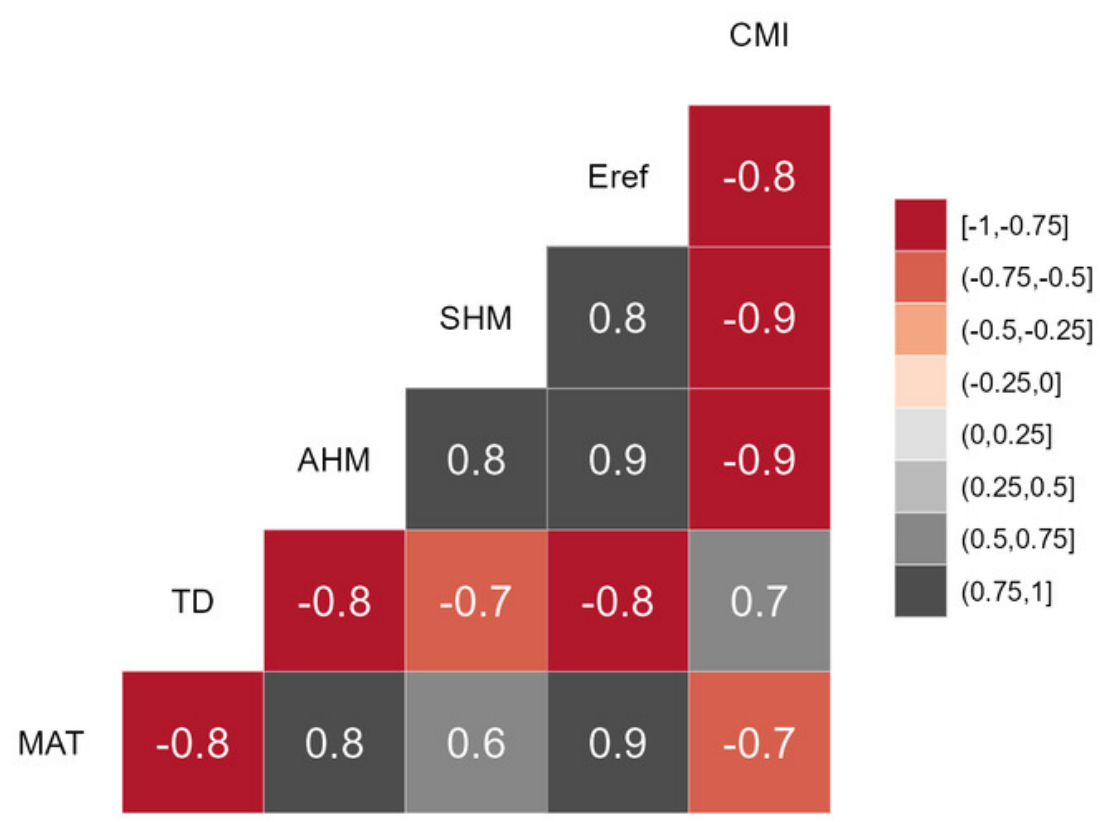

B

\section{X41284594}

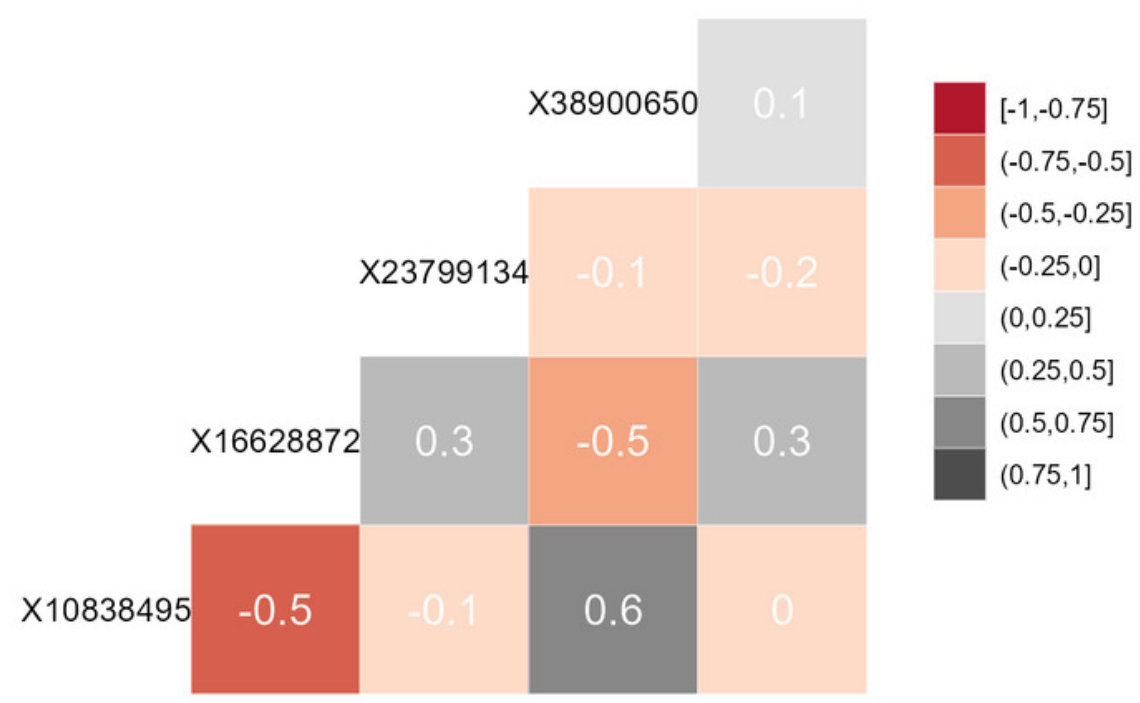


Figure 2

Ordination graphs.

(A) Principal Component Analysis (PCA) ordination graph for shifts in populations in environmental space. (B) Redundancy Analysis (RDA) ordination graph for shifts in populations with climate (a categorical variable with 'baseline' and 'future' as levels) as explanatory variable. 
A

Climate baseline $\triangle$ future

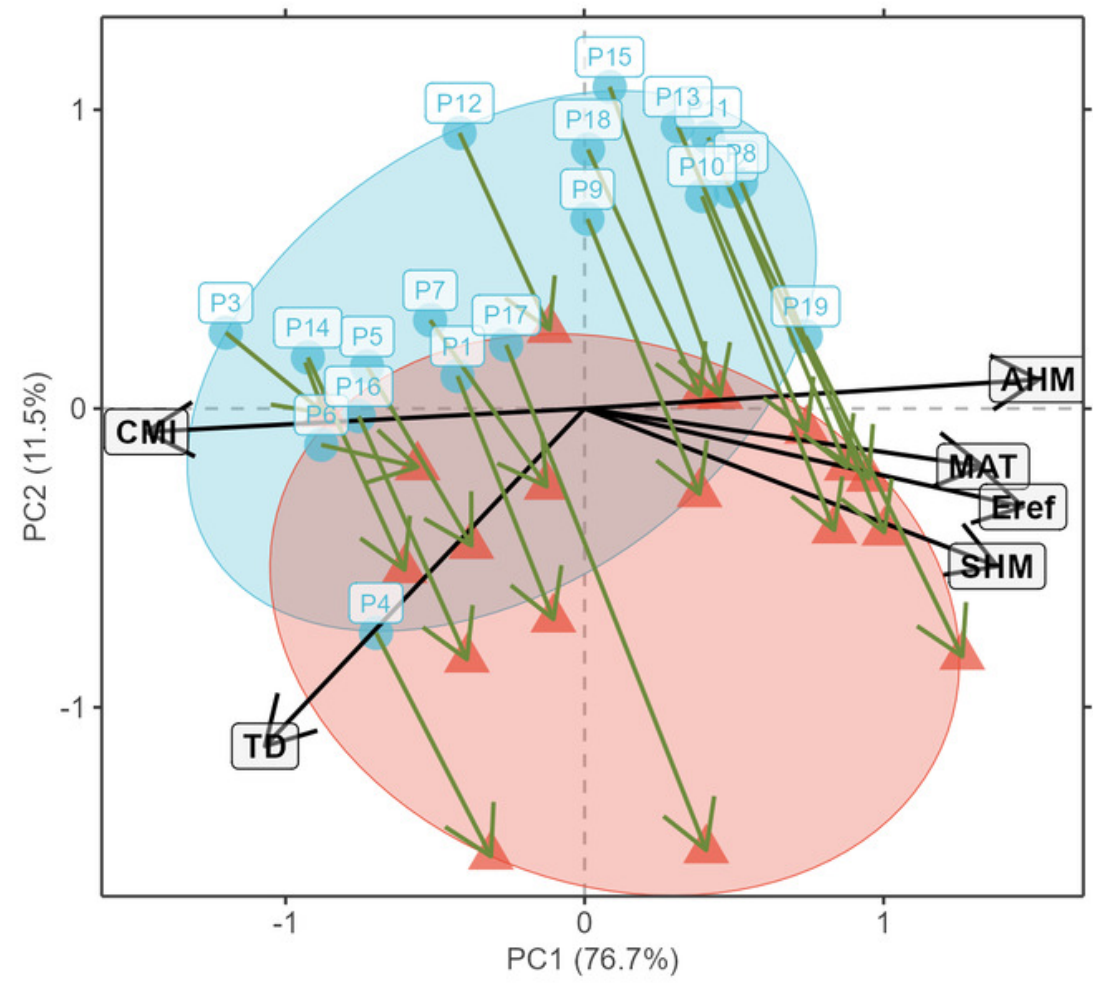

B

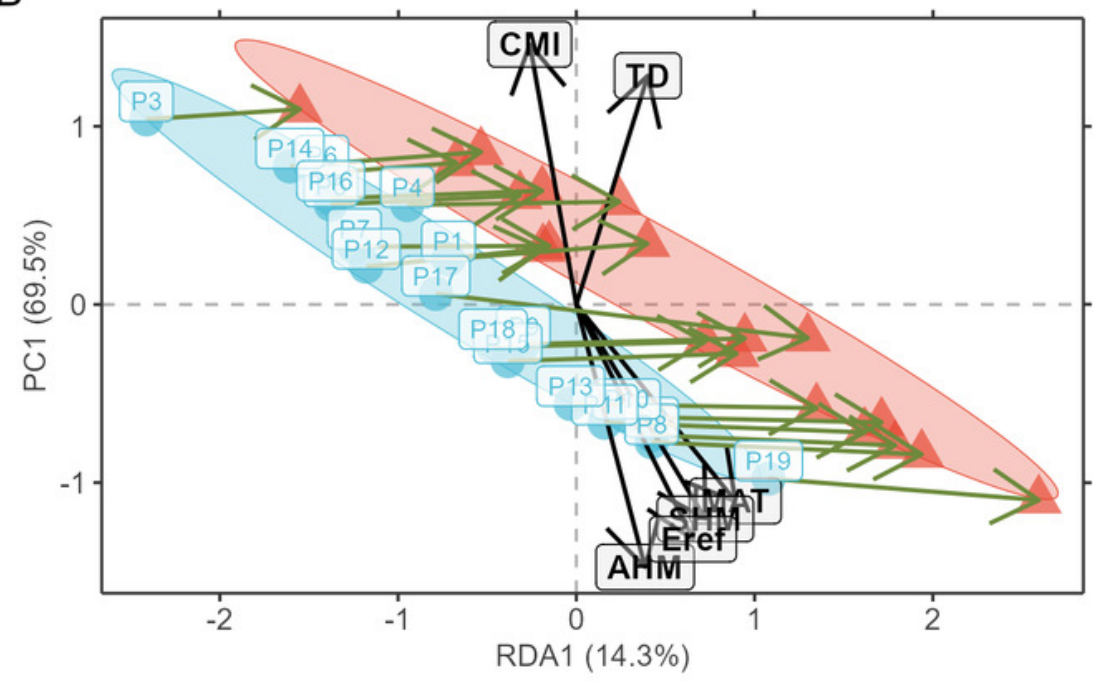


Figure 3

Plot of the actual frequency of the minor allele against the predicted frequency for the calibration data.

The 'olivegreen' reference lines indicate 1:1 (bold line), 1:1.05 and 1:0.95 (dashed lines) relationships. (A) Data for populations where a linear model explains more than $50 \%$ in allele frequencies. (B) Data for populations where a linear model explains less than $50 \%$ in allele frequencies. 


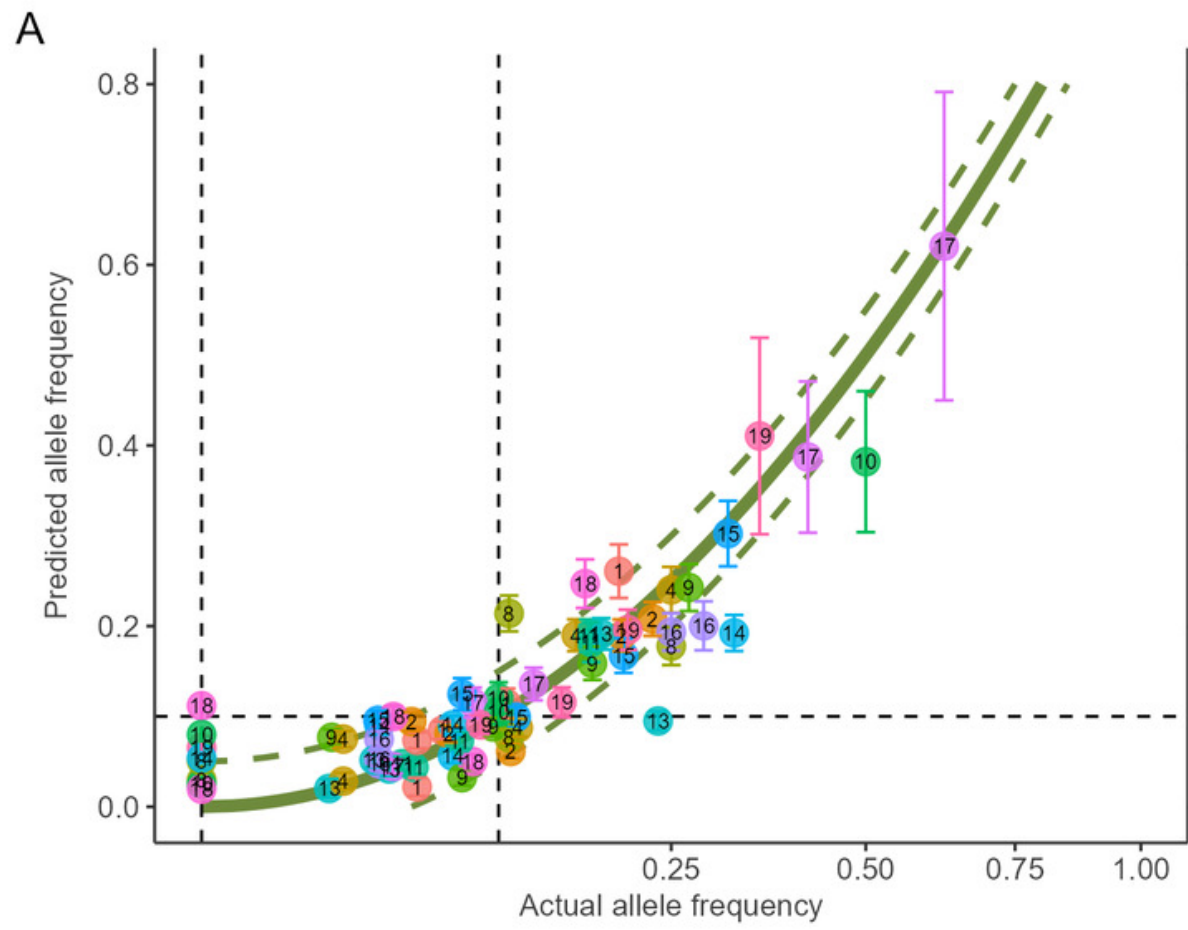

Population : $\mathrm{R}^{2}$

Chilliwack (P1) : 0.95

Columbia (P2) : 0.82

Harrison (P4) : 0.90

Nisqually (P8) : 0.54

Nooksack (P9) : 0.84

- Olympic_Penninsula (P10): 0.98

- Puyallup (P11) : 0.99

Skagit (P13) : 0.55

Skwawka (P14) : 0.90

Skykomish (P15): 0.89

Squamish (P16) : 0.98

Tahoe (P17) : 0.99

Vancouver I. East (P18) : 0.59

Willamette (P19) : 0.91

B

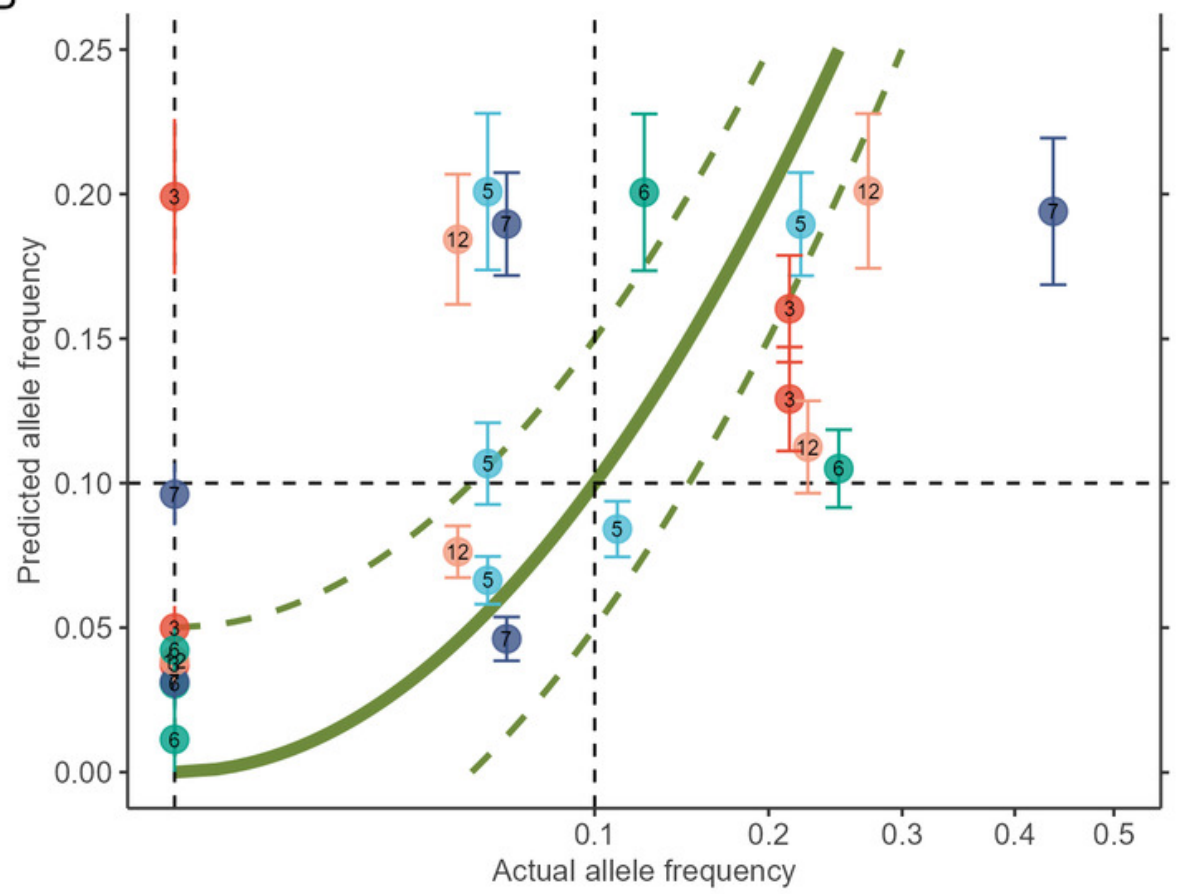

Population : $\mathrm{R}^{2}$

Dean (P3) : 0.15

Homathko (P5) : 0.17

Kitimat (P6) : 0.41

Klinaklini (P7) : 0.42

Salmon (P12) : 0.34 
Figure 4

Depiction of changes in minor allele frequencies via AlleleShift::shift.dot.ggplot.

Black circles reflect baseline frequencies, blue circles future frequencies and vertical lines the confidence interval 
Future change in allele frequencies $\_$decreasing $\_$increasing

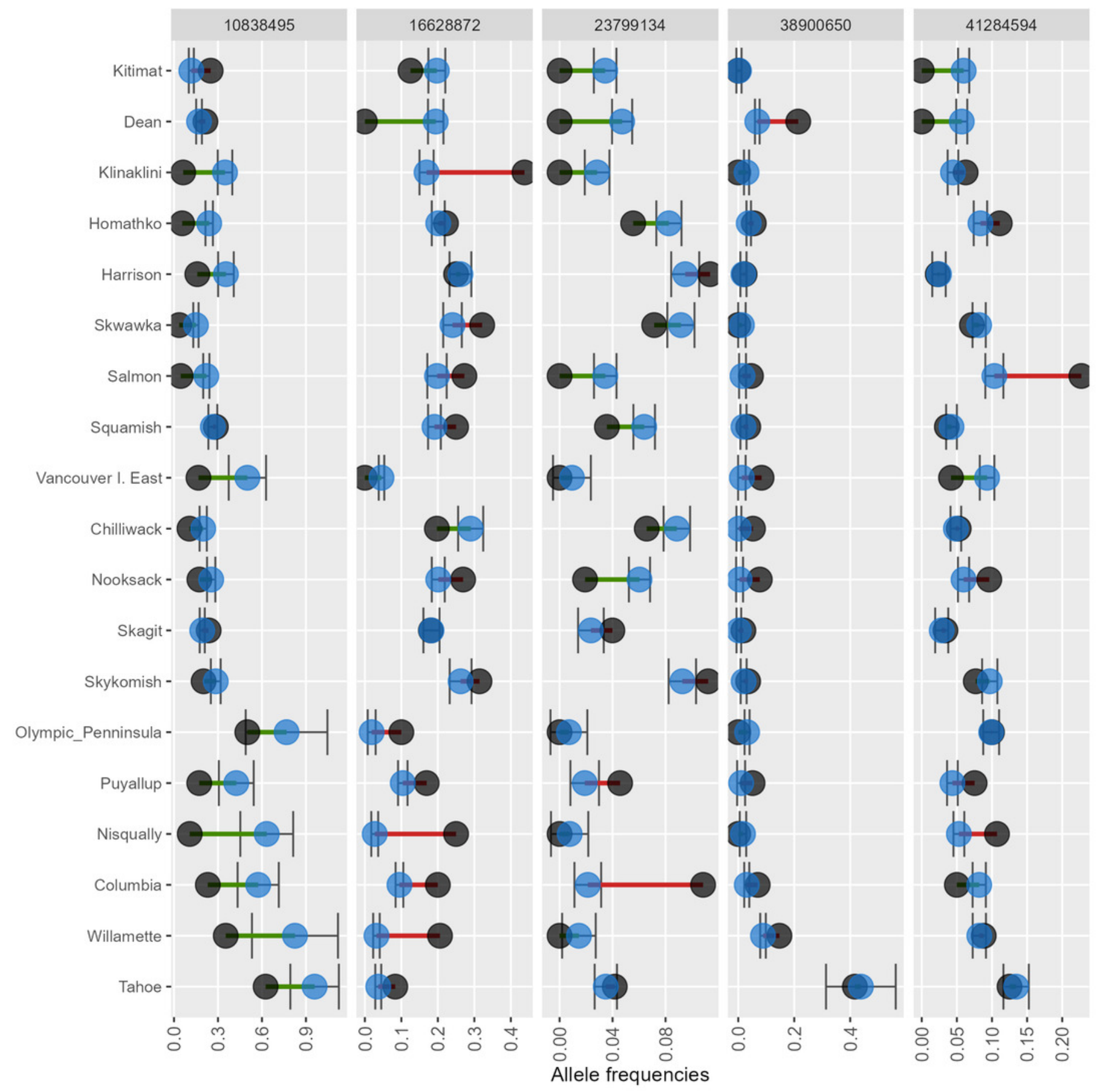


Figure 5

Depiction of changes in allele frequencies via AlleleShift::shift.pie.ggplot.

Columns on the left reflect baseline frequencies with frequency of the minor allele in black.

Columns on the right reflect future frequencies, with colour of the arc and colour of the central circle reflecting frequencies and trends (red $=$ decreasing, green $=$ increasing) of the minor allele. 


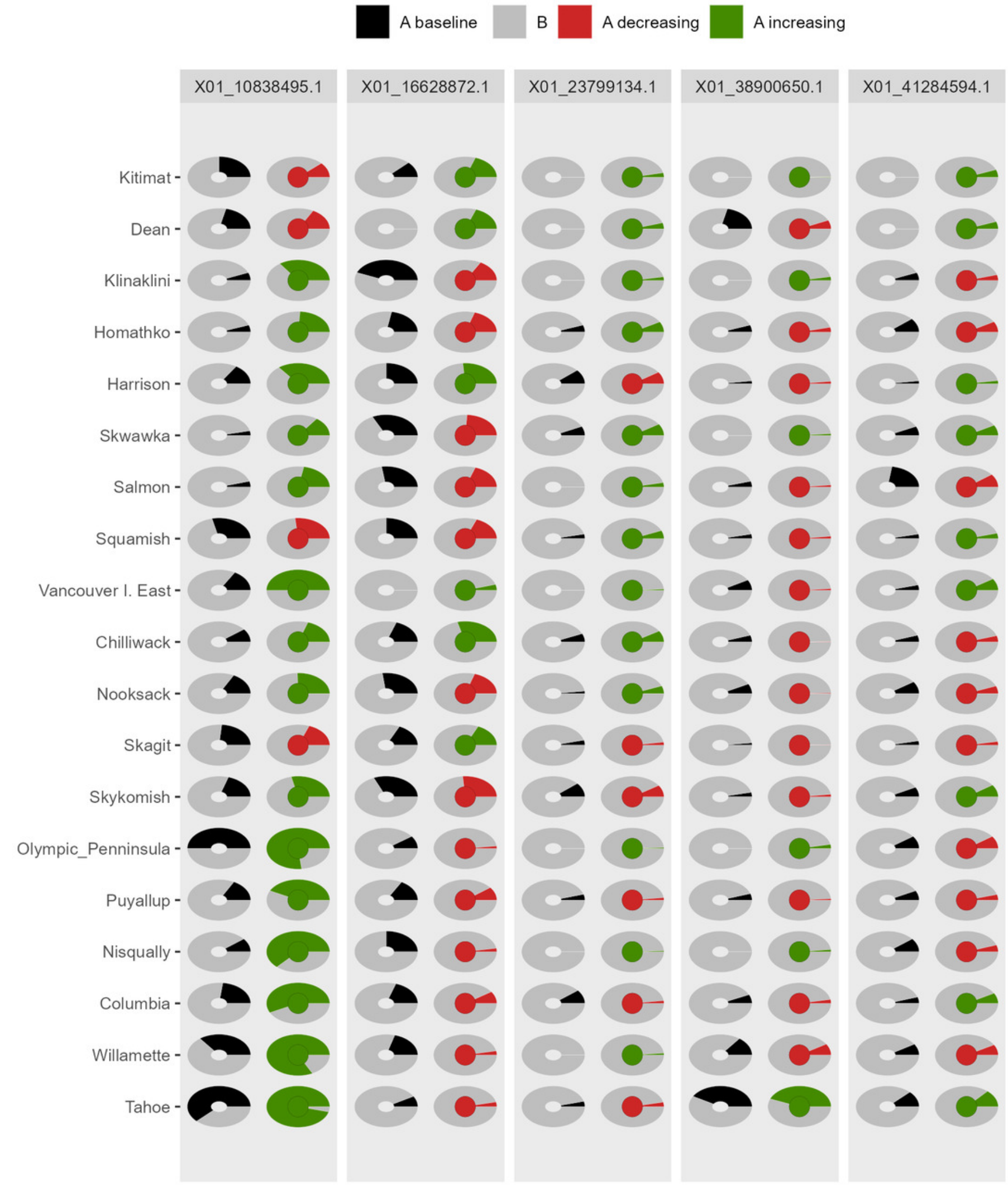




\section{Figure 6}

Depiction of changes in allele frequencies via AlleleShift::shift.moon.ggplot.

Columns on the left reflect baseline frequencies with frequency of the minor allele in white.

Columns on the right reflect future frequencies, with colour of the waxing moon and colour of the central circle reflecting frequencies and trends (red = decreasing, green $=$ increasing) of the minor allele. 


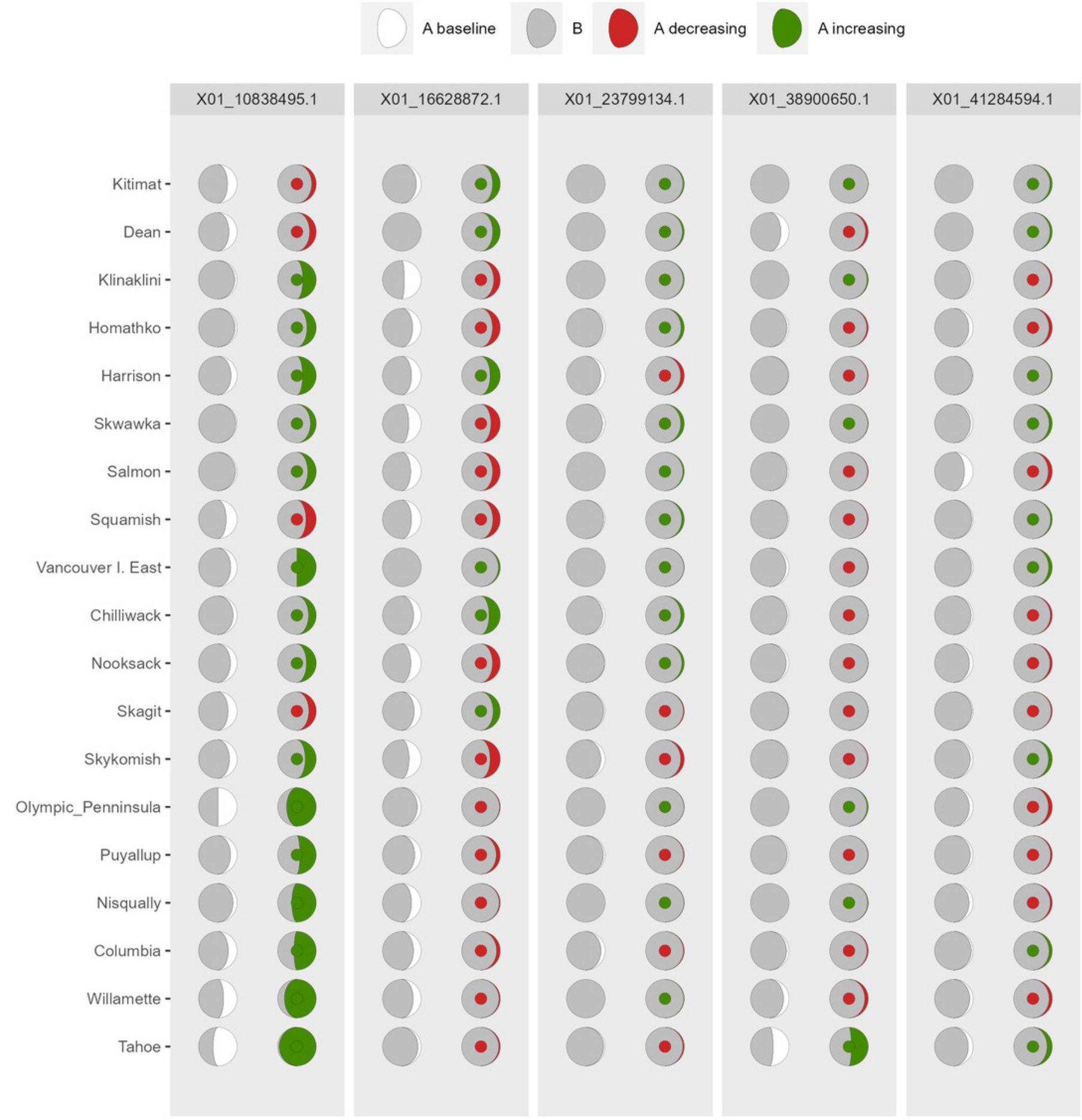




\section{Figure 7}

Depiction of changes in allele frequencies via AlleleShift::shift.waffle.ggplot.

Each 'waffle' has 100 'cells'. Columns on the left reflect baseline frequencies with frequency of the minor allele in black. Columns on the right reflect future frequencies of the minor allele, with colour indicating trends (red $=$ decreasing, green $=$ increasing) 


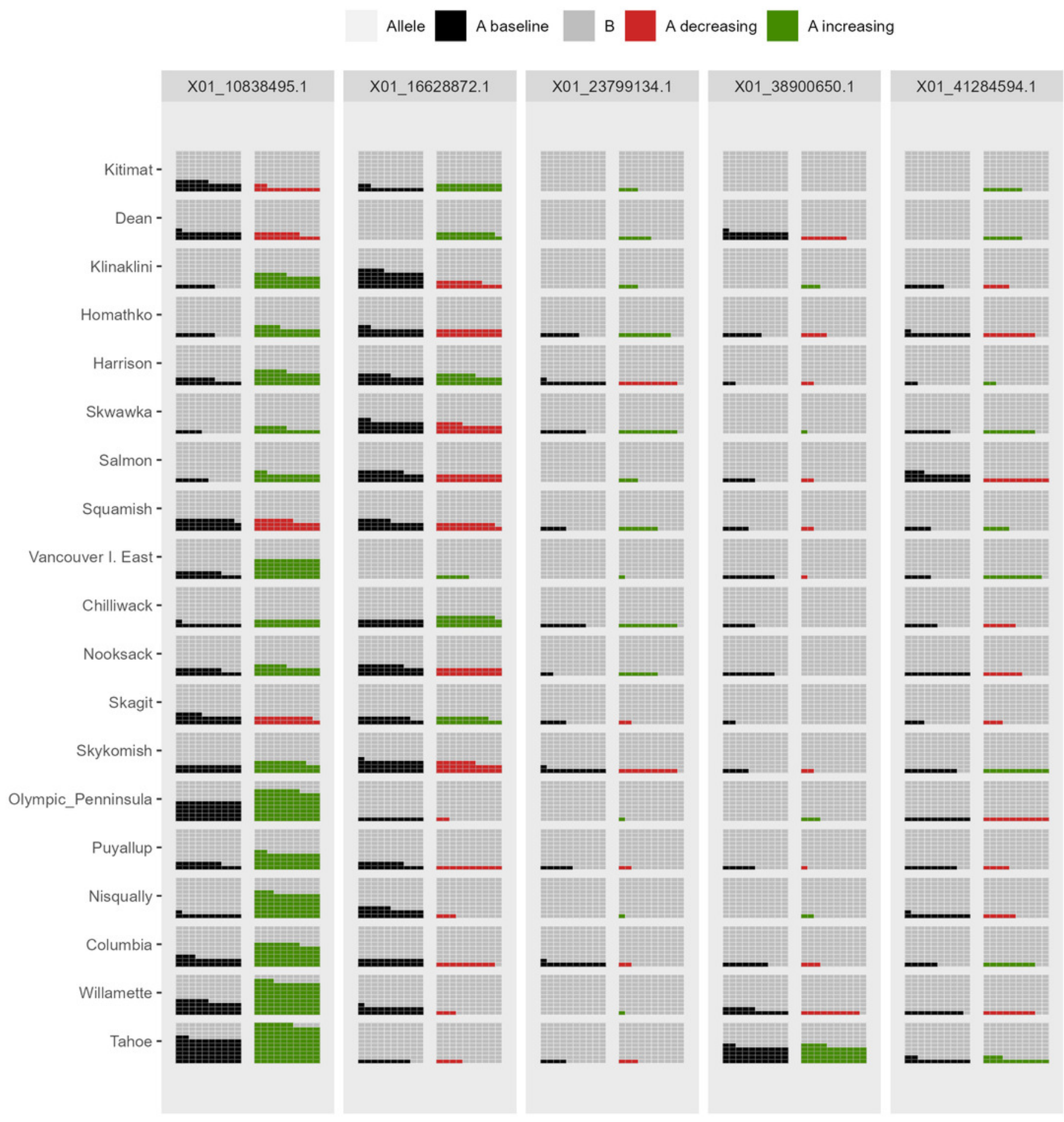




\section{Figure 8}

Depiction of changes in allele frequencies in geographical space via AlleleShift::shift.surf.ggplot.

(A) Frequencies in the baseline climate for the minor allele for locus 10838495. (B)

Frequencies in a future climate for the same allele as in (A). (C) Frequencies in the baseline climate for the minor allele for locus 23799134. (D) Frequencies in a future climate for the same allele as in (D). Sizes of circles reflect the frequencies for the populations. Colours for the future frequencies $(B, D)$ indicate trends (red $=$ decreasing, green $=$ increasing). 
A

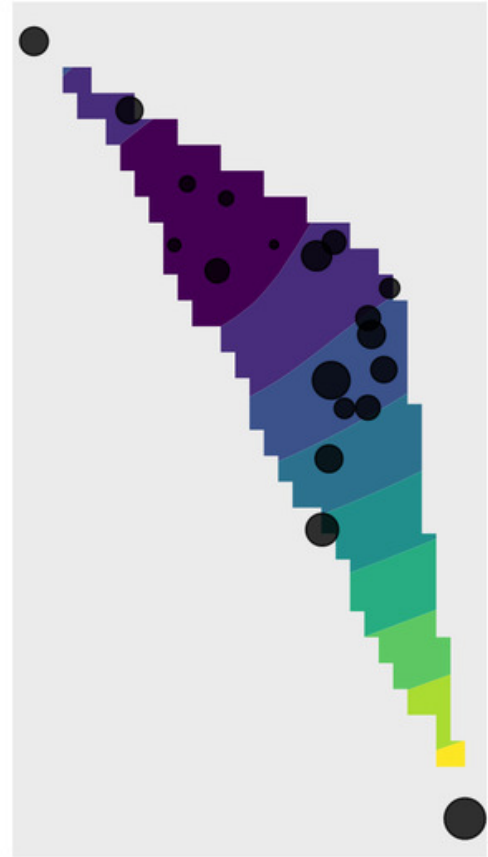

C

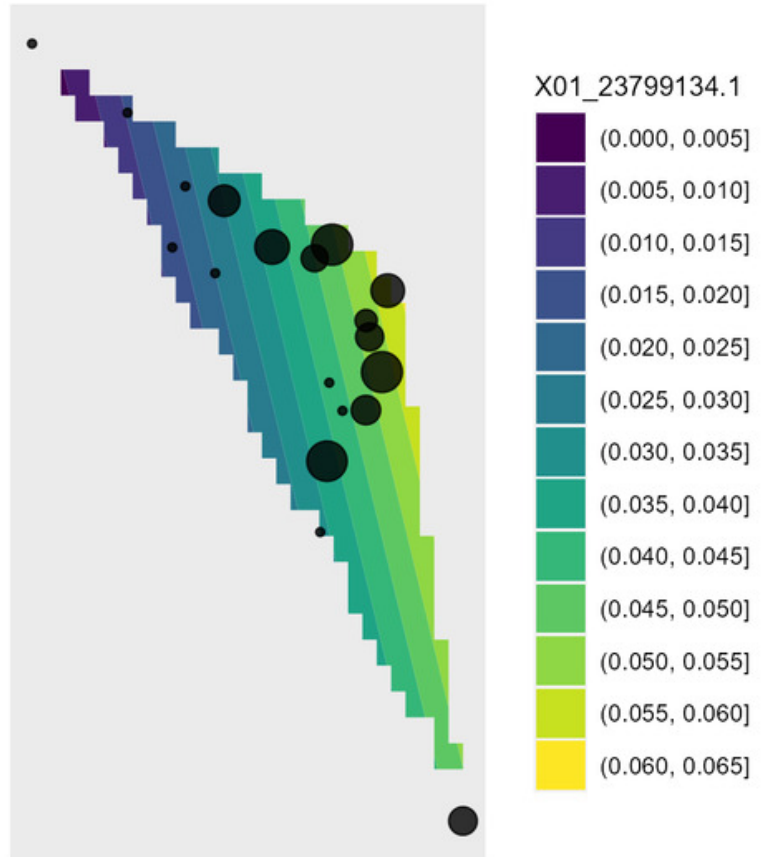

X01_10838495.1

$(0.10,0.15]$

$(0.15,0.20]$

$(0.20,0.25]$

$(0.25,0.30]$

$(0.30,0.35]$

$(0.35,0.40]$

$(0.40,0.45]$

$(0.45,0.50]$

$(0.50,0.55]$
B

D

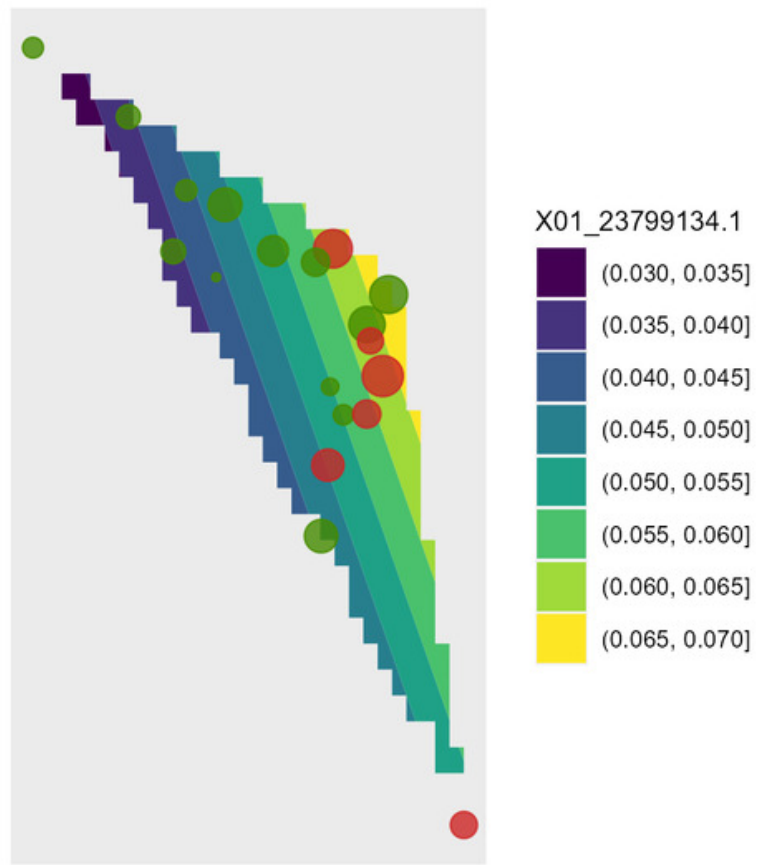

